\title{
Repulsive Casimir Forces Produced in Rectangular Cavities: Possible Measurements and Applications
}

\author{
A. Gusso \\ Departamento de Física, Universidade Federal do Paraná, C.P. 19044, 81531-990 Curitiba-PR, Brazil \\ and A. G. M. Schmidt \\ Departamento de Ciências Exatas e Tecnológicas, Universidade Estadual de Santa Cruz, CEP 45662-000, Ilhéus-BA, Brazil
}

Received on 10 August, 2005

\begin{abstract}
We perform a theoretical analysis of a setup intended to measure the repulsive (outward) Casimir forces predicted to exist inside of perfectly conducting rectangular cavities. We consider the roles of the conductivity of the real metals, of the temperature and surface roughness. The possible use of this repulsive force to reduce friction and wear in micro and nanoelectromechanical systems (MEMS and NEMS) is also considered.
\end{abstract}

Keywords: Micro and nanoelectromechanical systems; Casimir effect

\section{INTRODUCTION}

Casimir forces are a well known prediction of Quantum Field Theory, and result whenever the quantum vacuum is subject to constraints. The Casimir forces are one aspect of a broader subject usually referred to as Casimir effect. Presently, the Casimir effect finds applications not only in Quantum Field Theory, but also in Condensed Matter Physics, Atomic and Molecular Physics, Gravitation and Cosmology, and in Mathematical Physics [1-3], and its importance for practical applications is now becoming more widely appreciated $[4,5]$. Not withstanding its importance, the Casimir effect is elusive. The attractive Casimir forces (ACFs) predicted to exist between electrically neutral bodies were measured successfully only a few years ago [1]. Presently, the $\mathrm{ACF}$ between a sphere (or lens) above a flat disc covered with metals is claimed to be measured with an experimental relative error of approximately $0.27 \%$ at a $95 \%$ confidence level [6], and can be predicted with a theoretical uncertainty at the level of $1 \%$. The direct measurement of the Casimir force between two parallel conducting plates, the original setup studied by H. B. G. Casimir [7] in 1948, is even more challenging than for the sphere above a disc. For that reason it was accomplished only recently with a relatively poor precision of $15 \%$ [8].

The measurement of such attractive forces sheds some light on the question of the nature of the quantum electromagnetic vacuum. However, very important predictions based on the existence of the quantum vacuum have not received the same attention. This is the case of the repulsive Casimir forces (RCFs). Such repulsive forces (outward pressure on the walls) are predicted to exist inside of an empty sphere [9] and an empty rectangular cavity $[10,11]$ with perfectly conducting walls, for the case of Euclidean space. Such repulsive forces are probably the most striking example of the geometry dependent nature of the Casimir effect. However no experiment was performed to measure RCFs. Only a weak dependence on the geometry was tested measuring the force between a plate with small sinusoidal corrugations and a large sphere [12]. The measurement of the RCF would be one of the most important probes of the nature of the quantum vacuum with far reaching implications. Because RCFs have been predicted consistently by different quantum field theoretic techniques $[9-11,13]$ if they are proved not to exist the physicists will be faced with a new puzzle to be solved. Widely accepted Casimir energy renormalization and regularization procedures may need to be reviewed, as suggested by the only dissonant result presented in Ref. [14], were no RCFs are found for a rectangular piston.

The sign of the Casimir force is also predicted to depend upon electric and magnetic properties of materials. For instance, in Ref. [15] it is anticipated that a repulsive force will exist between two parallel plates if one is a perfect conductor and the other is perfectly permeable. More recently, a repulsive force between two parallel plates made from dielectric materials with nontrivial magnetic susceptibility was anticipated [16]. However, this effect, which could have interesting applications for MEMS and NEMS, has not been verified experimentally and no dielectric material exists satisfying the requirements on the values of the magnetic susceptibility.

In spite of the fact that RCFs are predicted for sphere and rectangular cavity with perfectly conducting walls, it is reasonable to expect, as for the case of parallel plates, that they will also be present inside cavities made from good conductors. For that reason, in this article we address the most important practical aspects to be taken into account in an experiment intended to measure the force exerted on one of the walls of a rectangular cavity: the finite conductivity and roughness of the walls and plate, and the temperature. The choice of rectangular cavity instead of the sphere is based primarily on the fact that the former could be most easily fabricated with the available techniques for the fabrication of MEMS and NEMS. We consider the experimental setup to measure the RCFs to be made of a series of microscopic metallic rectangular cavities arranged side by side, forming an array, with one of the walls open. The repulsive force is then measured by bringing near a plate with a flat metallic surface. The forces on the plate are then measured. The use of that can be measured most easily. This setup is presented schematically in Fig. 1(a). We note that a different setup was considered in Ref. [17] were a sphere 
is used instead of a flat plate. However, it has to be mentioned that in Ref. [17] it is considered the case in which the radius of the sphere is comparable to the cavity length, implying that the ends of the cavities are left essentially uncovered, and it is not clearly explained why in this case one still can expect the emergence of repulsive forces between the cavities and the sphere. Furthermore, the roughness of the walls and the role of the temperature are not considered in the analysis there presented. It is also not explained how the finite conductivity of the cavity walls and the sphere were taken into account in the calculation of the attractive and repulsive Casimir forces.

Its worth to mention that to analyze the flat platerectangular cavities configuration is specially relevant because the moveable pieces of MEMS and NEMS typically involve flat surfaces (for example, the rotary pieces in micromotors and gears), and it is natural to ask whether metallic rectangular cavities could be used to make such pieces to levitate or, at least, to have their weight or other undesirable forces partially compensated by a repulsive force. For that reason, following the analysis on the RCF measurement we present an analysis on the possible application of repulsive Casimir forces in MEMS and NEMS to circumvent the problems resulting from friction and wear.

\section{CASIMIR ENERGY AND FORCES}

In this section we argue that for a setup like that presented in Fig. 1 the resulting Casimir force on the plate is given by the sum of two independent contributions, namely, the RCF produced by the electromagnetic vacuum modes inside the cavity and the attractive force between the plate and the upper portion of the cavities.

The renormalized Casimir energy inside a rectangular cavity with perfectly conducting and perfectly smooth walls at zero temperature can be derived in various manners [1]. A simple expression suitable for numerical calculations was derived in [11], and in terms of the internal dimensions of the cavity $a_{1}, a_{2}$ and $a_{3}$ it reads

$$
\begin{aligned}
E_{C}= & -\hbar c \frac{a_{1} a_{2} a_{3}}{16 \pi^{2}} \sum_{l, m, n=-\infty}^{\infty}\left[\left(a_{1} l\right)^{2}+\left(a_{2} m\right)^{2}+\left(a_{3} n\right)^{2}\right]^{-2} \\
& +\hbar c \frac{\pi}{48}\left(\frac{1}{a_{1}}+\frac{1}{a_{2}}+\frac{1}{a_{3}}\right)
\end{aligned}
$$

The term with $n_{1}=n_{2}=n_{3}=0$ is to be omitted from the summation. From the principle of virtual work the force on the walls perpendicular to the direction of $a_{i}$ is simply

$$
F_{i}=-\frac{\partial E_{C}}{\partial a_{i}}
$$

and ranges from positive (outward) to negative (inward) depending on the relative sizes of $a_{1}, a_{2}$ and $a_{3}$. The Eqs. (1) and (2) allow one to search for the configuration of the cavity resulting into the strongest outward forces on the walls. The numerical analysis performed in Ref. [18], using the above expression for the energy, suggests that the forces $F_{2}$ and $F_{3}$ are larger (and positive) in a configuration satisfying $a_{1} \ll a_{2} \ll a_{3}$, corresponding to an elongated parallelepiped. For such a configuration $F_{1}$ is directed inward.

Fortunately, whenever $a_{1} \ll a_{2} \ll a_{3}$ for a rectangular cavity we can use a simple analytical expression for the Casimir energy $[1,10]$

$$
E_{C}=-\hbar c\left[\frac{\pi^{2} a_{2} a_{3}}{720 a_{1}^{3}}+\frac{\zeta_{R}(3)}{16 \pi} \frac{a_{3}}{a_{2}^{2}}-\frac{\pi}{48}\left(\frac{1}{a_{1}}+\frac{1}{a_{2}}\right)\right],
$$

where $\zeta_{R}$ denotes the Riemann zeta function. The expressions for the two outward forces are then calculated using Eq. (2) and the result is

$$
F_{2}=\hbar c\left[\frac{\pi^{2} a_{3}}{720 a_{1}^{3}}-\frac{\zeta_{R}(3)}{8 \pi} \frac{a_{3}}{a_{2}^{3}}+\frac{\pi}{48 a_{2}^{2}}\right]
$$

and

$$
F_{3}=\hbar c\left[\frac{\pi^{2} a_{2}}{720 a_{1}^{3}}+\frac{\zeta_{R}(3)}{16 \pi} \frac{1}{a_{2}^{2}}\right] .
$$

These formulas for the forces reproduce the results obtained from Eq. (1) to better than $1 \%$, and because the first term dominates over the others the forces are positive.

Therefore there are two possible configurations for our system. In one configuration elongated cavities with height $a_{2}$ are lying horizontally below the plate, like the cavity in Fig. 2(a), with the force exerted on the plate corresponding to $F_{2}$. In the other configuration the cavities are standing vertically below the plate, like the cavity in Fig. 2(b), with the force exerted on the plate corresponding to $F_{3}$. If there where no other forces acting on the plate, measuring the RCFs would be a relatively easy task. The plate could be brought near the open wall closing it completely, thus assuring the existence of the electromagnetic vacuum modes that lead to the Casimir energy Eq. (1). However, when the plate is close to the top of the walls there will be a resulting attractive force that can surpass the repulsive force. For that reason, in what follows we analyze which configuration is the more adequate for an experiment intended to measure RCFs, delivering the stronger repulsive force compared to the attractive forces between the plate and the cavity walls.

The repulsive forces exerted on the plate, $F_{2}$ or $F_{3}$, are expected to decrease with increasing $d$, the distance from the plate to the top of the walls [see Fig. 1(a)]. However, a detailed estimation of this decrease is beyond the scope of the present work. Instead we are going to assume in our calculations that the forces $F_{2}$ and $F_{3}$ do not depend on $d$. We expect this is a reasonable assumption whenever $d$ is sufficiently small in order not to disturb the electromagnetic vacuum modes that give the most important contributions to the Casimir energy. This expectation is based on the fact that in the case the aperture at the top of a cavity is smaller than $\lambda$ the transmission of the modes to outside the cavity is kept small [19]. Now the values of $\lambda$ that give the most important contribution to the Casimir energy for a cavity satisfying the condition $a_{1} \ll a_{2} \ll a_{3}$, 


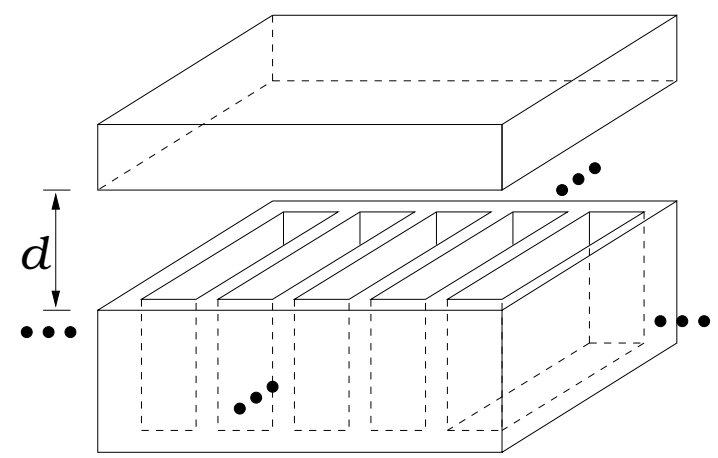

(a)

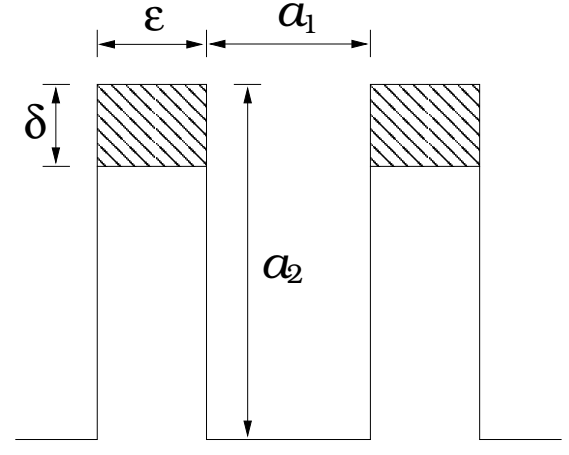

(b)

FIG. 1: (a) A view of the setup including the rectangular cavities and the plate. The dots denote the possibility of having more cavities arranged side by side. (b) The definitions of the lengths of the cavities and walls shown in a side view.

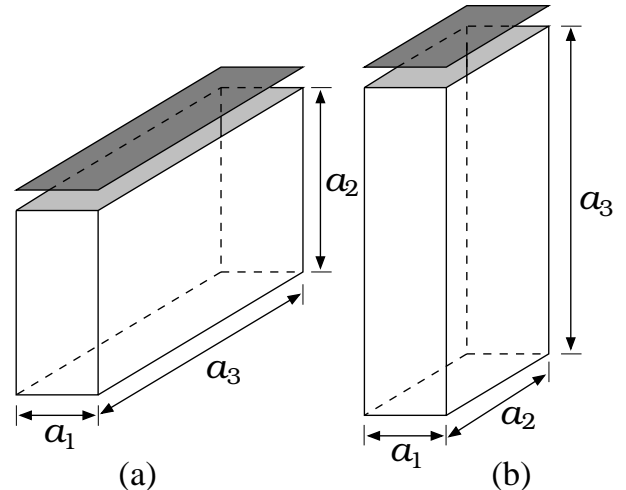

FIG. 2: (a) Cavity lying horizontally below a plate. (b) Cavity standing vertically below a plate. Plate is in dark gray and the open wall is indicated in light gray.

a geometry that closely resembles that of two parallel plates (more on that in Section III), are those of the order of the smallest edge $a_{1}$. Thus, it is reasonable to expect that $F_{2}$ and $F_{3}$ are constant up to $d \lesssim a_{1} / 2$. For $d \gtrsim a_{1} / 2$ the RCF will certainly decrease, and for that reason the relevant distance in an actual experiment is restricted to $d \lesssim a_{1}$.

Because $d \lesssim a_{1}$, and $a_{1} \ll a_{2}$ and $a_{3}$, we can separate the total Casimir force between the plate and the cavities into two components: the RCF, $F_{2}$ and $F_{3}$ and the ACF between the plate and the top of the walls. This conclusion is not as trivial as it may seen to be. If the plate were at relatively large distances from the cavities the Casimir energy for the platecavities system should be calculate from first principles considering the whole intricate geometry of the cavities. That means, the analysis should be similar to that carried on for periodically deformed objects in Ref. [20]. That would also be the case whether $a_{1} \sim a_{2}$, that means in the case of shallow cavities. For the deep cavities we are going to consider, only the interaction between the top of the walls and the plate is responsible for the attractive force.
Because of the nontrivial geometry involved, in order to calculate the ACF between the plate and the cavity walls we use the pairwise summation technique [21, 22]. This technique was shown to give reliable results for the Casimir force between bodies of arbitrary shape. For instance, for the force between a flat plate and a small body of arbitrary shape the maximum possible error was estimated to be $3.8 \%$ [21] when compared to the exact results obtained by quantum field theoretic techniques.

In the pairwise summation technique the Casimir energy is given by

$$
E_{C}^{\mathrm{pw}}=-\hbar c \Psi\left(\varepsilon_{20}\right)_{V_{1}}^{\mathrm{Z}} d^{3} r_{1}^{\mathrm{Z}}{ }_{V_{2}}^{3} d^{3} r_{2}\left|\mathbf{r}_{2}-\mathbf{r}_{1}\right|^{-7},
$$

where $V_{1}$ and $V_{2}$ are the volumes of the two interacting bodies, and $\Psi\left(\varepsilon_{20}\right)$ is a constant which depends on the materials on $V_{1}$ and $V_{2}$. This expression for the energy does not take into account the fact that the pairwise interaction between the atoms in the volumes $V_{1}$ and $V_{2}$ are actually screened by the surrounding atoms. In order to partially correct for this fact avoiding to overestimate the attractive forces we do not integrate over the entire volume of the walls and the plate. Instead we consider that interactions are only relevant up to a distance $\delta$ inside the metal. The resulting volumes of integration $V_{1}$ and $V_{2}$ are shown in Fig. 3, highlighted in light gray. Clearly, from Eq. (6) it is not important to define which volume corresponds to $V_{1}$ and $V_{2}$ since the variables are interchangeable. For the constant $\Psi\left(\varepsilon_{20}\right)$ we take its value in the limit of perfect conductors $\Psi\left(\varepsilon_{20}\right)=\pi / 24$, and introduce the corrections due to the finite conductivity later (Section III).

In order to get results that are independent of the exact number of cavities in the experimental setup, making the analysis more general, we employed a simple strategy. We note that the top of the walls, highlighted in light gray in Fig. 3(a), can be divided into a series of parallelepipeds. Therefore, because of the additivity of the Casimir energy in the context of the pairwise summation technique, the final Casimir energy between the plate and the walls is given by the sum of the individual energy of each segment (a parallelepiped) and the plate. The 

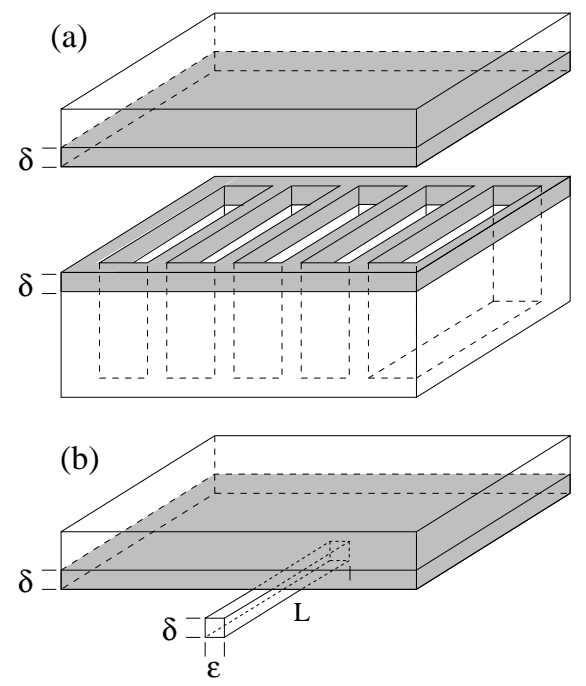

FIG. 3: (a) The relevant volumes of integration highlighted in light gray. (b) Parallelepiped below a large plate.

only restriction is that the plate must be sufficiently large to be considered infinite, making the calculations independent of the actual location of the different segments on the top of the walls. This condition can be easily fulfilled by a plate only slightly larger than the array of cavities because the Casimir energy decreases very rapidly with the increasing distance.

We evaluated $E_{C}^{\mathrm{pw}}$ analytically with the help of Mathematica [23] for a parallelepiped with dimensions given by $\varepsilon, \delta$ and arbitrary length $L$, below a plate with thickness $\delta$ and lateral dimensions that ensure that the final result is close enough to that for an infinitely large plate. This arrangement is depicted in Fig. 3(b). An energy per unity of area is obtained dividing $E_{C}^{\mathrm{pw}}$ by $\varepsilon \times L$. The final Casimir energy, including all the cavities is then obtained multiplying this energy per unit of area by the top surface of the walls, a procedure equivalent to summing over the different segments. Moreover, to make our analysis more general we assume that there is a sufficiently large number of cavities that we can calculate $E_{C}^{\mathrm{pw}}$ for one cavity and then simply multiply it by the total number of cavities. In such a case the contribution of the outermost walls that are partially disregarded are negligible. The area that enters in the calculation of $E_{C}^{\mathrm{pw}}$ is then the effective "attractive area" per cavity $S_{i}=\left(a_{1}+a_{i}+\varepsilon\right) \varepsilon$, where $i=2$ or 3 depending on whether the cavity is lying horizontally or standing vertically below the plate, respectively. The area of the plate under the action of the forces $F_{2,3}$ is $A_{2,3}=a_{1} \times a_{3,2}$. In what follows we always calculate the repulsive and attractive Casimir forces for only one cavity, but the results are actually valid for a large number of cavities as explained previously.

Similarly to the case of ACFs the RPCs are only appreciable when the dimensions of the cavity are in the micrometer range. This is the first practical aspect that has to be considered in any experiment. Presently, a structure like that in Fig. 1 can be made from metals like gold, nickel, copper, and aluminum with the smallest features with tens of nanometers, and structures like the cavity walls can be made with high aspect-ratios $[24,25]$. Consequently, the small dimensions of the cavities pose no problem if they are kept above a few tens of nanometers.

The pressures caused by $F_{2}$ and $F_{3}$, whenever $a_{1} \ll a_{2} \ll a_{3}$ goes with $1 / a_{1}^{2}$, as can be seen from Eqs. (4) and (5). Consequently, the smaller the $a_{1}$ the bigger the pressure, which is desirable. However, the smaller the $a_{1}$ the smaller the ratio $A_{i} / S_{i}$, for a given $\varepsilon$, therefore diminishing the ratio between the repulsive and the attractive forces. The lower limit on $a_{1}$ is then set by the lower practical limit on $\varepsilon$, because the walls must be thick enough to ensure good reflectivity to the electromagnetic modes inside the cavity. Such thickness is roughly determined by the penetration depth of the electromagnetic field $\delta_{0}=\lambda_{p} /(2 \pi)$, with $\lambda_{p}$ the plasma wavelength of the metal. For aluminum(gold) $\lambda_{p} \approx 107(136) \mathrm{nm}$ [27], implying $\delta_{0} \approx 17(22) \mathrm{nm}$. Now we note that the intensity of the incident electromagnetic wave a distance $x$ inside the metal decreases as $I=I_{0} \exp \left(-2 x / \delta_{0}\right)$. We can ensure almost no transmission of the electromagnetic waves by making $x_{\max }=\varepsilon \approx 2 \times \delta_{0}$. For that reason, we assume that the smallest possible thickness is $\varepsilon=30 \mathrm{~nm}$. For such an $\varepsilon$ the smallest $a_{1}$ is approximately $100 \mathrm{~nm}$. As we will see next, another reason not to take $a_{1}$ smaller than $100 \mathrm{~nm}$ is that for cavities made from real metals the RCFs are predicted to decrease significantly whenever the smaller side of the cavity is below approximately $100 \mathrm{~nm}$.

We now point out that the configuration of the rectangular cavity that can lead to the largest ratio between the repulsive and attractive forces on the plate is that of Fig. 2(a). This is so because in spite of the fact that both configurations can deliver the same outward pressures, in practice the ratio $F_{2} / S_{2}$ can be made greater (by one order of magnitude) than the ratio $F_{3} / S_{3}$. This results from the fact that the fabrication of a vertically standing rectangular cavity with thin walls much higher than $1 \mu \mathrm{m}$ would be very difficult. This implies that in general $a_{3} \lesssim$ $1 \mu \mathrm{m}$. Because for such a configuration $a_{2} \ll a_{3}$, the force $F_{3}$ is highly constrained [see Eq. (5)] compared to $F_{2}$, which can be made arbitrarily large since $a_{3}$ is not constrained. For that reason, in what follows we consider only the case the cavities are lying horizontally below the plate. This configuration is exactly the one depicted in Fig. 1(a).

\section{CONDUCTIVITY, ROUGHNESS AND TEMPERATURE CORRECTIONS}

The first correction to be taken into account here is that of finite conductivity, which alters both ACFs and RCFs. To this date finite conductivity corrections were calculated only for two simple geometries, namely, for two plane parallel plates and for a sphere above a disc [1,27]. Such calculations are quite involved, and similar calculations for a rectangular cavity and the interaction between the top of the walls and the plate would be even more demanding. Here, instead of calculating the corrections from first principles we adopt another strategy and use the results already obtained for plane parallel plates. 
In order to justify this approach we note that the Casimir energy for a rectangular cavity satisfying $a_{1} \ll a_{2} \ll a_{3}$ is approximately that in a region with dimensions $a_{2} \times a_{3}$ between two infinite parallel plates separated by a distance $a_{1}$

$$
E_{C}^{0}=-\hbar c \frac{\pi^{2} a_{2} a_{3}}{720 a_{1}^{3}},
$$

as can be inferred from Eq. (3). That means the modes inside the cavity are approximately the same as those between parallel plates a distance $a_{1}$ apart. Hence it is reasonable to assume that the corrections to the energy and $F_{1}$ for the rectangular cavity are adequately described by those to the energy and force between parallel metallic plates. In the notation of Ref. [27] we write, $E_{C}=\eta_{E}\left(a_{1}\right) E_{C}^{0}$ and $F_{1}=\eta_{F}\left(a_{1}\right) F_{1}^{0}$, where $E_{C}^{0}$ and $F_{1}^{0}$ are the energy and force for a cavity with perfectly conducting walls. The functions $\eta_{E, F}(x)$ are the correction factors that range from approximately 1 at large separations to approximately 0 at the shortest distances. These factors depend upon the materials on the walls through their frequency dependent dielectric functions. In our analysis we modeled the dielectric functions using the plasma model as done in Ref. [27]. In this context, since $\eta_{E}$ does not depend upon $a_{2}$ the correction to the force $F_{2}$ is the same as that for the energy. However, in order to be conservative we assume that the correction to $F_{2}$ is the same as that for $F_{1}\left(\eta_{F}\right.$ is slightly smaller than $\eta_{E}$ ). In conclusion, we assume that $F_{2}=\eta_{F}\left(a_{1}\right) F_{2}^{0}$, where $\eta_{F}\left(a_{1}\right)$ is plotted in Fig. 1 of Ref. [27] and $F_{2}^{0}$ is given by Eq. (4).

We have chosen to analyze two rectangular cavities with dimensions that have a good commitment with the need for strong RCF, to be approximate by parallel plates, and to have reasonable aspect-ratios to meet the requirements of available fabrication techniques, namely

$$
a_{1}=0.1 \mu \mathrm{m}, a_{2}=0.5 \mu \mathrm{m}, a_{3}=5 \mu \mathrm{m}
$$

and

$$
a_{1}=0.2 \mu \mathrm{m}, a_{2}=1 \mu \mathrm{m}, a_{3}=5 \mu \mathrm{m} .
$$

We consider cavities made from aluminum, for its excellent reflectivity in a wide range of frequencies, and gold, a metal widely employed for the fabrication of MEMS. For the cavity with $a_{1}=0.1(0.2) \mu \mathrm{m}: F_{2}=2.1(0.27) \mathrm{pN}$; the pressure is $P_{2}=4.2(0.27) \mathrm{N} \mathrm{m}^{-2}$; for aluminum $\eta_{F}\left(a_{1}\right)=0.50(0.68)$; and for gold $\eta_{F}\left(a_{1}\right)=0.44(0.62)$. We stress that the energy inside the cavity for perfectly conducting walls differs from that for parallel plates with area $a_{2} \times a_{3}$ by just $0.8(3) \%$, therefore justifying our assumptions.

The finite conductivity correction for the ACF was taken to be the same as that for parallel plates, and the force obtained from the use of Eq. (6) is simply multiplied by $\eta_{F}(d)$. This is certainly a good approximation whenever the separation $d$ is small compared to $\varepsilon$, because in such a case the top of the walls and the plate form a system resembling two parallel plates. For $d$ comparable or greater than $\varepsilon$ we do not expect that this approximation fails completely. This expectation relies on the fact that for larger distances the correction factor is nearly 1 and vary at a relatively slow pace, consequently, it is less important. Another consequence of the finite conductivity is a rapid decay of electromagnetic fields inside the metal. It was for that reason that we considered a finite $\delta$ in the calculation of the ACF. Based on the values of $\delta_{0}$ for aluminum and gold we assume $\delta=50 \mathrm{~nm}$.

The second correction to the forces that we consider is that of surface roughness. What is relevant here is the stochastic roughness in both the cavity walls and the plate resulting from the fabrication process. As we did for the finite conductivity corrections we use the results already derived for the case of two parallel plates. The corrected energy inside the cavity can be obtained from the expression for the corrected force between two plates in Ref. [1] by simply integrating on the separation, resulting in

$$
E_{C}^{\text {roughness }}=E_{C}^{0}\left[1+4\left(\frac{\delta_{\text {disp }}}{a_{1}}\right)^{2}+60\left(\frac{\delta_{\text {disp }}}{a_{1}}\right)^{4}\right],
$$

where $\delta_{\text {disp }}$ is the dispersion (roughly the amplitude) of the stochastic roughness. In this approximation there is no dependence of the roughness correction on $a_{2}$ and $a_{3}$. Consequently, the force $F_{2}$ is corrected by exactly the same factor as the energy. To keep the corrections below the 1(5)\% level it is required that $\delta_{\text {disp }} / a_{1} \lesssim 0.049(0.10)$. That means for a cavity with $a_{1}=100 \mathrm{~nm}$ that the imperfections on the walls can be as large as 5(10) nm. Presently, by means of electron beam lithography a precision in the level of $1.3 \mathrm{~nm}$ has been obtained for the fabrication of MEMS and NEMS [28]. However, most usual techniques are not that accurate and a precision at the level of $10 \mathrm{~nm}$ is most likely to be found in an experiment [24]. As a first approximation the ACF could also receive the same correction expressed in Eq. (10).

Finally we address the role of temperature. An expression for the Casimir energy inside a rectangular cavity with finite temperature was derived in Ref. [26]. It corresponds to adding the following terms to the energy in Eq. (1)

$$
\begin{aligned}
E_{C}^{\mathrm{temp}} & =\hbar c\left(-\frac{\pi^{2} a_{1} a_{2} a_{3}}{45 \beta^{4}}+\frac{\pi}{12 \beta^{2}}\left(a_{1}+a_{2}+a_{3}\right)\right. \\
& -\frac{a_{1} a_{2} a_{3}}{\pi^{2}} \sum_{l, m, n, p=1}^{\infty} \frac{1}{\left[\left(a_{1} l\right)^{2}+\left(a_{2} m\right)^{2}+\left(a_{3} n\right)^{2}+\left(\frac{\beta}{2} p\right)^{2}\right]^{2}} \\
& \left.+\frac{1}{\pi} \sum_{l, p=1}^{\infty}\left\{\frac{a_{1}}{\left[4\left(a_{1} l\right)^{2}+(\beta p)^{2}\right]}+\frac{a_{2}}{\left[4\left(a_{2} l\right)^{2}+(\beta p)^{2}\right]}+\frac{a_{3}}{\left[4\left(a_{3} l\right)^{2}+(\beta p)^{2}\right]}\right\}\right),
\end{aligned}
$$


where $\beta=\hbar c / k_{B} T$, with $k_{B}$ the Boltzmann's constant and $T$ the absolute temperature. For the cavity with $a_{1}=0.1(0.2) \mu \mathrm{m}$ at a temperature of $300 \mathrm{~K}$ the energy decreases significantly by $1.1(4.7) \%$, while the force $F_{2}$ decreases just $0.08(0.2) \%$. The corrections are still very small at higher temperatures.

\section{FORCE MEASUREMENT}

In the present analysis of force measurement we disregard both the temperature and roughness corrections. The former because the correction to the force is always much smaller than the expected experimental accuracy on the force measurement; of the order of a few percent in any realistic scenario. The later because it could be made suitably small depending on the fabrication technique. Yet, we consider the most important correction, that of the finite conductivity, that reduce the repulsive force produced by the cavity by half of its original value and the attractive forces by even greater factors [27]. Besides being the most important correction, greatly exceeding the expected corrections due to roughness, the conductivity will depend upon the material the cavity and the plate are made from and only marginally on the fabrication technique. In this sense, the corrections due to conductivity are universal, and do not depend upon the specific fabrication technique that will be employed, henceforth justifying the present theoretical analysis.

As already mentioned in Section II in the analysis we did not model the expected decrease on the repulsive force as a function of the separation $d$. However, it is reasonable to assume that for small separations the repulsive force can be well described by Eq. (4). For small separations we mean $d$ small compared to $a_{1}$, because it is the smaller cavity dimension that, after all, determines the the smaller frequencies allowed inside the cavity. For small $d$ we can expect a small perturbation on the modes inside the cavity in a large range of frequencies, henceforth ensuring the existence of the repulsive force.

The most important information for an experiment designed to measure RCFs is the ratio between the repulsive and attractive forces as a function of the separation $d$. In Figs. 4(a) and 4(b) we present exactly this ratio for the cavities with $a_{1}=0.1 \mu \mathrm{m}$ and $0.2 \mu \mathrm{m}$, respectively. The results are for the cavity and the plate made from gold, however essentially the same results are obtained for aluminum. We considered four different $\varepsilon$, from the smallest possible value to one that could be most easily obtained by the presently available fabrication techniques.

What we can infer from Fig. 4 is the smallness of the repulsive force compared to the attractive one in the range of distances at which our calculations are more reliable and precise $\left(d \lesssim a_{1} / 2\right)$. The ratios are larger for the cavity with $a_{1}=200$ $\mathrm{nm}$, and for $d=a_{1} / 2=100 \mathrm{~nm}$ the RCF amounts to $30 \%$ of the ACF. For the cavity with $a_{1}=100 \mathrm{~nm}$ the RCF amounts to only $10 \%$ at $d=a_{1} / 2$. As a consequence of the smallness of the ratios $F_{\text {rep }} / F_{\text {at }}$, any measurement of the force exerted on the top plate has to be very precise. For the static measurement of the force on the top plate a precise knowledge of the
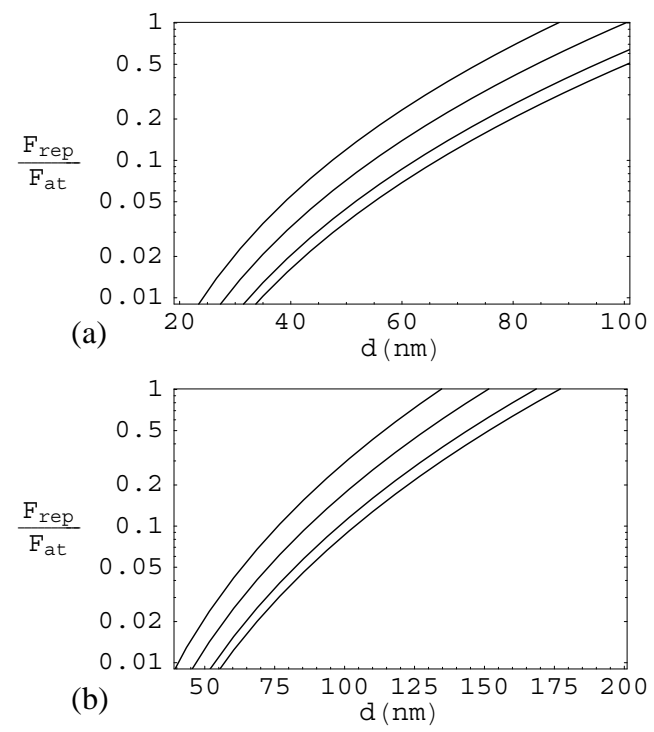

FIG. 4: The ratio between repulsive $\left(F_{\text {rep }}\right)$ and attractive $\left(F_{\text {at }}\right)$ Casimir forces for a rectangular cavity as a function of the separation $d$. From the upper to the lower curve $\varepsilon=30,50,80$, and $100 \mathrm{~nm}$. Cavity dimensions in (a) given by Eq. (8) and in (b) by Eq. (9).

separation $d$ is also required. This fact can be illustrated by the ratio between the sum of the attractive force at the actual position and the repulsive force and the attractive force at the distance $d$ as determined from the experiment,

$$
\frac{F^{*}}{F_{\text {at }}}=\frac{F_{\text {at }}(d+\Delta)+F_{\text {rep }}}{F_{\text {at }}(d)},
$$

where $\Delta$ represents the relative displacement to the measured distance due to the uncertainties. The curves for this ratio are presented in Fig. 5 for the two cavities and for $\Delta=0, \pm 1$ and $\pm 3 \mathrm{~nm}$. The upper(lower) curves are for negative(positive) $\Delta$. We note that even for $\Delta= \pm 1 \mathrm{~nm}$ the errors are in the range $5-15 \%$ and are of the order of the force the experiment intends to measure (see Fig. 4). Consequently, the distance has to be measured with an accuracy better than $1 \mathrm{~nm}$. In order to estimate the required accuracy we note that for a nominal separation $d=50 \mathrm{~nm}$, an inaccuracy of $0.2 \mathrm{~nm}$ implies an uncertainty in the force measurement of approximately $\pm 1.5 \%$ for both cavities, which is acceptable.

Our analysis leads to the conclusion that very stringent requirements have to be satisfied by the experimental setup in order to allow for an adequate measurement of the RCF in a rectangular cavity. Such requirements surpass considerably those for the experiments already carried out for the measurement of ACFs $[1,6,8]$.

\section{APPLICATIONS}

As already mentioned in section I, repulsive forces could have interesting applications in MEMS and NEMS. In fact, 

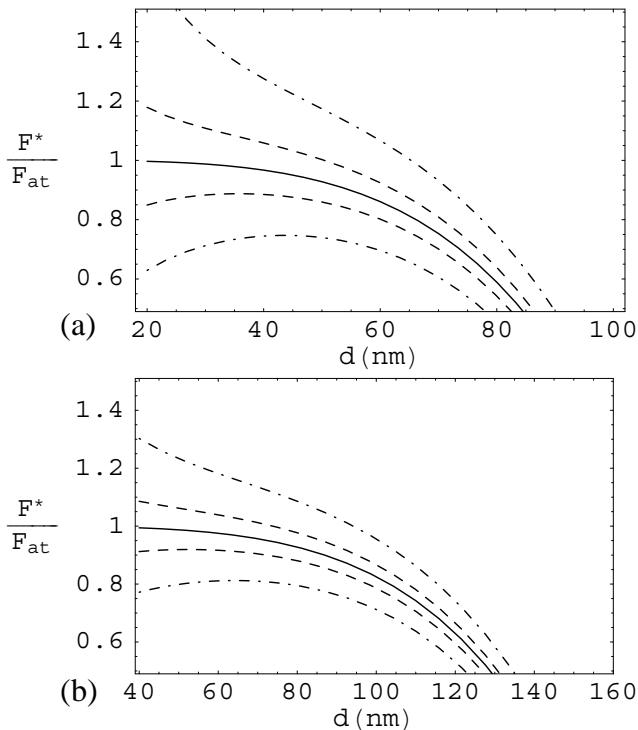

FIG. 5: Curves for the ratio defined in Eq. (12) for $\Delta=0$ (continuous), $\Delta= \pm 1 \mathrm{~nm}$ (dashed) and $\Delta= \pm 3 \mathrm{~nm}$ (dot-dashed).Cavity dimensions in (a) given by Eq. (8) and in (b) by Eq. (9).

such forces could be the solution for the problems that are presently imposing severe restrictions on the functioning of MEMS with moveable parts, namely, friction and wear [29]. The forces caused by friction are usually very large at small scales [30] when compared to the forces that can be delivered by the available driven systems in, e.g., micromotors and microactuators. Usually friction obeys Amonton's law (frictional force depends linearly on the load through the coefficient of friction), however, at small scales friction turns out to be proportional to the contact area between the surfaces [30]. For systems sufficiently large to obey Amonton's law, repulsive forces could be used to reduce the load. For instance, the rotary piece of a micromotor or gear (usually with the shape of a disc) could be lifted by a bottom force that could partially or completely compensates for its weight. This force could be the RCF predicted in Ref. [16] or, as we propose here, the force produced by a set of rectangular cavities placed beneath the rotary piece of the micromotor or gears. The first option requires the use of suitable materials that presently are not available, and is still a matter of debate whether such forces could actually exist $[31,32]$. The second option, the use of cavities beneath the moveable pieces, could be a simple solution whenever these pieces were made from metals or could at least be covered with a thin metal layer. For smaller systems, where the load does not play the most important role in the resulting frictional force, the repulsive forces could be used in the same way to reduce the effective weight that has to be sustained by the rotating pivots or bearings. Consequently, the pivots and bearings could possibly be smaller, leading to a reduction in the frictional force and wear. Such a reduction is highly desirable since wear is the most important source of failure in MEMS , limiting their continuous operation lifetime
TABLE I: The distances $d_{0}, d_{1}$ and $d_{10}$ as defined on the text for cavities made from $\mathrm{Al}(\mathrm{Au})$, and dimensions given in Eq. (8).

\begin{tabular}{cccc}
\hline \hline$\varepsilon(\mathrm{nm})$ & $d_{0}(\mathrm{~nm})$ & $d_{1}(\mathrm{~nm})$ & $d_{10}(\mathrm{~nm})$ \\
\hline 30 & $88.2(88.1)$ & $89.4(89.5)$ & $107(112)$ \\
50 & $100(100)$ & $102(102)$ & $126(135)$ \\
\hline \hline
\end{tabular}

TABLE II: The distances $d_{0}, d_{0.1}$ and $d_{1}$ as defined on the text for cavities made from $\mathrm{Al}(\mathrm{Au})$, and dimensions given in Eq. (9).

\begin{tabular}{cccc}
\hline \hline$\varepsilon(\mathrm{nm})$ & $d_{0}(\mathrm{~nm})$ & $d_{0.1}(\mathrm{~nm})$ & $d_{1}(\mathrm{~nm})$ \\
\hline 30 & $135(134)$ & $137(136)$ & $163(166)$ \\
50 & $152(151)$ & $154(153)$ & $186(192)$ \\
\hline \hline
\end{tabular}

to be of the order of seconds or minutes rather than hours or days [29].

To estimate the capability of the repulsive force produced by the rectangular cavities to compensate for the weight of the moveable parts of MEMS and NEMS we determined the distance $d_{0}$ at which the RCF equates the ACF and the distances required to the repulsive force to equate the $\mathrm{ACF}$ added to the weight of a plate made from a metal with an intermediate density $\rho=8.9 \mathrm{~g} \mathrm{~cm}^{-3}$ (similar to that of nickel and cooper) and thickness of $1 \mu \mathrm{m}$ and $10 \mu \mathrm{m}$ denoted $d_{1}$ and $d_{10}$, respectively. At this point we have to note that structures with thickness ranging from $0.1 \mu \mathrm{m}$ up to $10 \mu \mathrm{m}$ are usually employed in the fabrication of parts of MEMS and NEMS [25] even when the other dimensions of these parts are of the order of a few millimeters [33]. We present in Table I $d_{0}, d_{1}$ and $d_{10}$ for the cavity with $a_{1}=0.1 \mu \mathrm{m}$, made from aluminum and gold, and for the thickness of the walls $\varepsilon=30 \mathrm{~nm}$ and $50 \mathrm{~nm}$. In Table II the results are presented for the cavity with $a_{1}=0.2 \mu \mathrm{m}$. In this case, because the repulsive force is not strong enough to equate the weight of a plate $10 \mu \mathrm{m}$ thick, we present the distance $d_{0.1}$ required to sustain a plate with thickness of 0.1 $\mu \mathrm{m}$.

In order to better understand the implications of the results presented in Tables I and II we have to remember the fact that the RCF produced by the cavity on the plate is expected to decrease with the separation $d$. Actually, from simple wave propagation arguments, the change on the force is expected to depend upon the ratio $d / a_{1}$. Consequently, we can expect a smaller correction (smaller decrease) to the force due to the separation for the cavity for which the ratio $d / a_{1}$ is smaller. We now note that for $\varepsilon=30 \mathrm{~nm} d_{0}\left(d_{1}\right)$ corresponds to $88(89) \%$ and $68(82) \%$ of the cavity width for $a_{1}=0.1 \mu \mathrm{m}$ and $0.2 \mu \mathrm{m}$, respectively. For that reason the cavity with $a_{1}=0.2 \mu \mathrm{m}$ is the most adequate for investigations concerning the reduction of friction and wear. Because $d_{0.1}$ is only slightly larger than $d_{0}$, levitation of thin metallic plates caused by RCF is also likely to occur.

We also suggest the use of an artifice in order to reduce further the distances at which repulsive forces could counterbalance the attractive forces: thiner walls with short height built on top of the cavity walls. Thiner walls may assure enough reflectivity for the electromagnetic modes inside the cavity with- 
TABLE III: The distances $d_{0}, d_{1}$ and $d_{10}$ as defined on the text for cavities made from $\mathrm{Al}(\mathrm{Au})$, with the dimensions given in Eq. (8), and with additional top walls.

\begin{tabular}{cccc}
\hline \hline$\varepsilon(\mathrm{nm})$ & $d_{0}(\mathrm{~nm})$ & $d_{1}(\mathrm{~nm})$ & $d_{10}(\mathrm{~nm})$ \\
\hline 30 & $78.4(78.3)$ & $79.7(79.7)$ & $96.6(102)$ \\
50 & $82.0(82.0)$ & $83.5(83.8)$ & $107(116)$ \\
\hline \hline
\end{tabular}

TABLE IV: The distances $d_{0}, d_{0.1}$ and $d_{1}$ as defined on the text for cavities made from $\mathrm{Al}(\mathrm{Au})$, with the dimensions given in Eq. (9), and with additional top walls.

\begin{tabular}{cccc}
\hline \hline$\varepsilon(\mathrm{nm})$ & $d_{0}(\mathrm{~nm})$ & $d_{0.1}(\mathrm{~nm})$ & $d_{1}(\mathrm{~nm})$ \\
\hline 30 & $125(124)$ & $127(126)$ & $153(157)$ \\
50 & $132(132)$ & $134(134)$ & $166(173)$ \\
\hline \hline
\end{tabular}

out further disturbing the modes if they are kept sufficiently short. The small aspect-ratio further facilitates their fabrication. For instance, if the top walls were $15 \mathrm{~nm}$ thick and 45 $\mathrm{nm}$ high the distances between the top of these walls and the plate are predicted to be those presented in Tables III and IV. In calculating those distances we summed over the contributions from the original wall and the additional top wall. The contribution of the original wall is small as can be seen from the similarity between the results for $\varepsilon=30 \mathrm{~nm}$ and $50 \mathrm{~nm}$ in Tables III and IV as compared to the results in Tables I and II that differ considerably.

It is clear that the introduction of the top walls can considerably reduce the required separations. If the top walls can be made thiner and taller without further disturbing the modes inside the cavity is a subject that deserves further theoretical and experimental investigation. Triangular structures are also worth of investigation. Anyhow, for the shorter distances thus obtained the assumption of a constant RCF as $d$ varies is more reliable, and therefore the results are self-consistent.

\section{FINAL DISCUSSION AND CONCLUSIONS}

In this article we presented a realistic analysis of a setup intended to measure the repulsive forces resulting from the geometrical constraints imposed on the quantum electromagnetic vacuum. For realistic we mean that the nonideality of the cavity was taken into account in the calculation of the RCF as well as the unavoidable ACF. We took advantage of the similarity between a rectangular cavity satisfying the condition $a_{1} \ll a_{2} \ll a_{3}$ and two plane parallel plates, considerably simplifying the analysis. The results thus obtained are expected to be a very good description of the reality for small ratios $d / a_{1}$ and still reliable when the ratio is around 0.5 .

¿From the results presented in section IV we conclude that for the smaller separations at which our approach is more precise, attractive forces are always considerably greater than the attainable repulsive forces. This fact poses severe requirements for the experiment. For separations larger than approx- imately $a_{1} / 2$ a reduction of the repulsive force is expected and the curves in Figs. 4 and 5 are no longer precise. However, these curves indicate that even under the more optimistic assumption that the decrease in the RCF is small and that the reliability of our results extends to larger separations, the measurement may be difficult, unless the cavity walls are sufficiently thin. That this is specially true for the case the cavity has $a_{1}=0.1 \mu \mathrm{m}$, can be seen from the fact that for $\varepsilon=100 \mathrm{~nm}$, at a separation $d=a_{1}=0.1 \mu \mathrm{m}$ the repulsive force amounts to only $50 \%$ of the attractive force. Fortunately, we have a better situation for the case of a larger cavity since the RCF equates the ACF at shorter distances, as can be seen in Fig. 4(b).

It is worth to mention that there seems to be no advantage on the use of cavities with $a_{1}$ much greater than $200 \mathrm{~nm}$. The reason for that is the fact that for cavities with larger $a_{1}$ the ratio $F_{\text {rep }} / F_{\text {at }}$ is essentially the same as that for $a_{1}=200 \mathrm{~nm}$ when plotted as a function of $d / a_{1}$. Nevertheless, the RCF and ACF decrease significantly, possibly making its measurement less precise. This fact has to be considered in the design of any actual experiment.

The use of the plasma model in the calculation of the finite conductivity corrections results in correction factors $\eta_{F}$ that are from $2 \%$ to $10 \%$ smaller than those predicted using the tabulated data for the dielectric functions of aluminum and gold for distances around $100 \mathrm{~nm}$ [27]. This fact along with our conservative assumption that the force $F_{2}$ is corrected by the factor $\eta_{F}$ for the force $F_{1}$, may imply that the actual repulsive forces delivered by the cavities in an experiment are greater than the ones we predicted here by at most $20 \%$. Such an increase in the force does not significantly changes our results because of the strong dependence of the attractive forces on the separation $d$. More precisely, the distances would decrease no more than $5 \%$.

The most obvious use of the RCF in MEMS and NEMS is to levitate structures as we proposed here, preventing friction and wear. However, the applicability of such forces is conditioned to the actual decrease of the RCF with the separation between the cavities and the upper (plate-like) structure. As already mentioned the determination of the actual repulsive force with the distance is beyond the scope of the present work, and is expected to be quite involved, specially in the case that the finite conductivity of the walls are taken into account. Nonetheless, the results presented in section V, based on the extrapolation of a constant RCF to larger separations, indicate that the RCF produced by the rectangular cavity is potentially useful and the importance of the reduction of wear and friction in MEMS and NEMS makes it worth of further investigation.

\section{Acknowledgments}

The authors gratefully acknowledge $\mathrm{CNPq}$ for research fellowships. A. G. M. Schmidt was also partially supported by Fundação de Amparo à Pesquisa do Estado da Bahia (FAPESB). A. Gusso is thankful to I. A. Hümmelgen, D. H. Mosca and E. S. Silveira for useful conversations. 
[1] M. Bordag, U. Mohideen, and V. M. Mostepanenko, Phys. Rep. 353, 1 (2001).

[2] G. Plunien, B. Müller, and W. Greiner, Phys. Rep. 134, 87 (1986).

[3] P. W. Milonni, The Quantum vacuum: An Introduction to Quantum Electrodynamics, (Academic Press, New York, 1994).

[4] E. Buks and M. L. Roukes, Nature 419, 119 (2002).

[5] H. J. de los Santos, Proc. IEEE 91, 1907 (2003); G. J. Maclay, H. Fearn, and P. W. Milonni, Eur. J. Phys. 22, 463 (2001).

[6] R. S. Decca et al., Phys. Rev. D 68, 116003 (2003).

[7] H. B. G. Casimir, Proc. K. Ned. Akad. Wet. 51, 793 (1948).

[8] G. Bressi, G. Carugno, R. Onofrio, and G. Ruoso, Phys. Rev. Lett. 88, 041804 (2002).

[9] T. H. Boyer, Phys. Rev. 174, 1764 (1968).

[10] S. G. Mamaev and N. N. Trunov, Theor. Math. Phys. (USA) 38, 228 (1979); Sov. Phys. J. 22, 966 (1979).

[11] J. Ambjørn and S. Wolfram, Ann. Phys. (N. Y. ) 147, 1 (1983).

[12] Anushree Roy and U. Mohideen, Phys. Rev. Lett. 82, 4380 (1999).

[13] S. Hacyan, R. Jáuregui, and C. Villarreal, Phys. Rev. A 47, 4204 (1993).

[14] M. P. Hertzberg, R. L. Jaffe, M. Kardar, and A. Schardicchio, quant-ph/0509071.

[15] T. H. Boyer, Phys. Rev. A 9, 2078 (1974); F. C. Santos, A. Tenório, and A. C. Tort, Phys. Rev. D 60, 105022 (1999).

[16] O. Kenneth, I. Klich, A. Mann, and M. Revzen, Phys. Rev. Lett. 89, 033001 (2002).

[17] J. Maclay et al., published as AIAA/ASME/SAE/ASEE 37th Joint Propulsion Conference, Salt Lake City, July 8, 2001 (available at http: / / www . quantumfields. com); G. J. Maclay and J. Hammer, in Proc. of the 7th International Conference on Squeezed States and Uncertainty Relations edited by D. Han,
Y. S. Kim, B. E. A. Saleh, A. V. Sergienko, and M. C. Teich, available only in electronic format at http://www.physics . umd.edu/rgroups/ep/yskim/boston/boston.html

[18] G. J. Maclay, Phys. Rev. A 61, 052110 (2000).

[19] P. M. Morse and P. J. Rubenstein, Phys. Rev. 54, 895 (1938); Min Li et al., IEEE Trans. Eletromag. Compat. 39, 225 (1997)

[20] T. Emig, Europhys. Lett. , 62, 466 (2003); Phys. Rev. A 67, $022114(2003)$

[21] V. M. Mostepanenko and I. Yu. Sokolov, Sov. Phys. Dokl. (USA) 33, 140 (1988).

[22] M. Bordag, G. L. Klimchitskaya, and V. M. Mostepanenko, Mod. Phys. Lett. 19, 2515 (1994); Int. J. Mod. Phys. A 10, 2661 (1995).

[23] S. Wolfram, The Mathematica Book, 4th ed., (Wolfram Media/Cambridge University Press, 1999)

[24] C. R. K. Marrian and D. M. Tennant, J. Vac. Sci. Technol. A 21, S207 (2003).

[25] H. G. Graighead, Science 290, 1532 (2000).

[26] F. C. Santos and A. C. Tort, Phys. Lett. B 482, 323 (2000).

[27] A. Lambrecht and S. Reynaud, Eur. Phys. J. D 8, 309 (2000).

[28] J. T. Hastings, Feng Zhang, and Henry I. Smith, J. Vac. Sci. Technol. B 21, 2650 (2003).

[29] S. L. Miller et al., Microelectron. Reliab. 39, 1229 (1999); J. A. Williams, Wear 251, 965 (2001); W. Merlijn van Spengen, Microelectron. Reliab. 43, 1049 (2003).

[30] Weiyuan Wang et al., Sens. Actuators A 97-98, 486 (2002).

[31] D. Iannuzzi and F. Capasso, Phys. Rev. Lett. 91, 029101 (2003).

[32] C. Henkel and K. Joulain, quant-ph/0407153.

[33] See available information at http://mems.sandia.gov/ scripts/index.asp. 